\title{
Prevalence of Metabolic Syndrome in Patients With Type 2 Diabetes Mellitus in a Tertiary Care Hospital
}

\author{
Dipesh Shakya ${ }^{1}$ and Vijay $K C^{2}$
}

${ }^{1}$ Department of Neurology, Kathmandu Medical College Teaching Hospital, Sinamangal, Kathmandu, Nepal. ${ }^{2}$ Department of Internal Medicine, Kathmandu Medical College Teaching Hospital, Sinamangal, Kathmandu, Nepal.

\begin{abstract}
Introduction: Diabetes mellitus is associated with various metabolic disorders, which leads to the progression of the disease and its complications. The aim of the study is to find out prevalence of metabolic syndrome and its association with the components and demographic variables.

Methods: This cross-sectional study was conducted among diabetes mellitus patients at a Tertiary level teaching hospital over a period of one year (April 2015 to March 2016). The study involved the use of a questionnaire to obtain information on diabetes by performing anthropometric measurements and corroborating it with respective blood samples collected for the measurement of biochemical parameters, fasting blood glucose and lipid profile. Metabolic syndrome was defined according to the current guidelines, revised in 2005 by the National Heart, Lung and Blood Institute and the American Heart Association. Statistical Package for the Social Sciences Version 20 was used as a tool for statistical analysis.

Results: This cross-sectional study involved 200 type 2 diabetes mellitus patients. The prevalence of metabolic syndrome was $71 \%$ in the studied Nepalese population. Central obesity (77.5\%) and hypertension (76.8\%) were the commonest risk factors. It was followed by dyslipidemia among which $65.5 \%$ had high triglyceride level and $50.7 \%$ had low level of high density lipoprotein cholesterol. Higher prevalence of metabolic syndrome was seen in male patients with type 2 diabetes which accounted for 84 (73.3\%). Three components of metabolic syndrome were seen among $70(35 \%)$ and five components among 29 (14.5\%) patients. Among the patients who were studied, the age group 45 to 54,55 to 64 and 65 to 74 years had $14(20 \%), 20(28.6 \%)$ and $19(27.1 \%)$ of more than three components of metabolic syndrome respectively.

Conclusions: The prevalence of metabolic syndrome was $71 \%$ among the patients with type 2 diabetes, where high prevalence was seen among males. In our study central obesity and hypertension along with increasing age were the most common component causing metabolic syndrome.
\end{abstract}

Key words: Diabetes mellitus; Metabolic syndrome.

Correspondence: Dipesh Shakya, Department of Neurology, Kathmandu Medical College Teaching Hospital, Sinamangal, Kathmandu, Nepal. Email: shakyadeepu20@hotmail.com

DOI: http://dx.doi.org/10.3126/mjsbh.v18i2.22207

Submitted on: 2019-01-07

Accepted on: 2019-06-12 


\section{INTRODUCTION}

The metabolic syndrome, as defined by the National Cholesterol Education Program Adult Treatment Panel III (NCEP-ATP III) criteria, is a constellation of fasting lipids/lipoproteins, waist circumference, glucose and blood pressure abnormalities. This has been associated with increasing risk for developing cardiovascular disease (CVD) and diabetes. ${ }^{1,2}$ Using the data from the National Health and Nutrition Examination Survey 1999 to 2002 database, $34.5 \%$ of participants met Adult Treatment Panel (ATP) III criteria for the metabolic syndrome compared to $22 \%$ in the third National Health and Nutrition Examination Survey (NHANES III, 1988 to 1994). ${ }^{3,4}$ Type 2 diabetes and metabolic syndrome has been regarded as a disease of adults for a long time. $^{5}$

Type 2 diabetes accounts for $90-95 \%$ of diabetes. It is usually associated with family history, older age, obesity, physical inactivity, or a personal history of gestational diabetes. ${ }^{6}$ Obesity (particularly abdominal obesity), is associated with resistance to insulin on peripheral glucose and fatty acid utilisation, often leading to type 2 diabetes mellitus. Insulin resistance associated hyperinsulinemia, hyperglycemia, and adipocyte cytokines (adipokines) may lead to vascular endothelial dysfunction, an abnormal lipid profile, hypertension, and vascular inflammation, all of which promote the development of atherosclerotic cardiovascular disease (CVD) ${ }^{7-9} \mathrm{CVD}$ and diabetes both have complications which may eventually handicap the patient. Since the components are modifiable the disease can be prevented significantly if proper interventions are taken on time.

However, metabolic syndrome is becoming increasingly common among the younger age groups which warns the need to correct the components promptly and with combined approach. The aim of the present study is to find out the prevalence, common components and demography of the metabolic syndrome in type 2 diabetes mellitus among Nepalese population.

\section{METHODS}

This cross-sectional prospective analytical study was held at a tertiary level teaching hospital in Kathmandu from April 2015 to March 2016. All those patients who were diagnosed with type 2 diabetes mellitus irrespective of the anti-diabetic medication visiting inpatient and outpatient service were included in the study. Sample size was calculated using consecutive sampling technique.

The study involved the use of a questionnaire to obtain history and information regarding diabetes mellitus undertaking anthropometric measurements such as waist circumference, height and weight. Blood samples were collected for the measurement of biochemical parameters such as fasting blood glucose and lipid profile.

Metabolic syndrome was defined according to the revised 2005 National Heart, Lung, and Blood Institute (NHLBI) and the American Heart Association (AHA) ${ }^{10}$ i.e.

- Fasting glucose $\geq 100 \mathrm{mg} / \mathrm{dL}$ (or receiving drug therapy for hyperglycemia)

- Blood pressure $\geq 130 / 85 \mathrm{~mm}$ of $\mathrm{Hg}$ (or receiving drug therapy for hypertension)

- Triglycerides $\geq 150 \mathrm{mg} / \mathrm{dL}$ (or receiving drug therapy for hypertriglyceridemia)

- HDL-C $<40 \mathrm{mg} / \mathrm{dL}$ in men or $<50 \mathrm{mg} / \mathrm{dL}$ in women (or receiving drug therapy for reduced HDL-C)

- Waist circumference for Asian: $\geq 90 \mathrm{~cm} \mathrm{(35}$ inche) in men or $\geq 80 \mathrm{~cm}$ (32 inch) in women

Metabolic syndrome was diagnosed when a patient had at least three of the above five conditions.

The analysis was done using Statistical Package for the Social Sciences (SPSS) software Version 20. Simple descriptive analysis and Chi-square test was used to determine the risk factors. Institutional ethical clearance was obtained and informed consent was taken from the patients. 


\section{RESULTS}

The present study included 200 patients with type 2 diabetes mellitus among which males were $56.5 \%$ and females were $43.5 \%$ with mean age of 57.56 years. Metabolic syndrome was diagnosed in 142 (71\%) type 2 diabetic patients based on the revised guidelines of National Heart Lung and Blood Institute (NHLBI) and the American Heart Association (AHA). Among the patients with metabolic syndrome, 84 were males and 58 were females.

Based on the distribution of prevalence of components of metabolic syndrome, according to the revised NHLBI and AHA, the levels of waist circumference (WC), blood pressure (BP), triglycerides (TGR) were high in $77.5 \%, 76.8 \%$ and $65.5 \%$ of the samples respectively. In contrast, high density lipoprotein-cholesterol (HDL-C) was low in only $50.7 \%$ of the patients (Table 1 ).

The most common risk factors were central obesity, as indicated by increased WC (77.5\%) and high blood pressure $(76.8 \%)$ followed by hypertriglyceridemia (65.5\%). Regarding the distribution of patients according to the number of components of metabolic syndrome, 70 (35\%) and 29 (14.5\%) participants had three and five components respectively (Figure 1). It was also observed that the patients in age groups $45-54,55-64$ and $65-74$

Table 1. Percentage of the parameters within the metabolic syndrome

\begin{tabular}{|l|c|c|}
\hline Parameters & \multicolumn{2}{|c|}{ Metabolic syndrome } \\
\hline & $\begin{array}{c}\text { Yes } \\
\mathrm{n}(\%)\end{array}$ & $\begin{array}{c}\text { No } \\
\mathrm{n}(\%)\end{array}$ \\
\hline Blood pressure $\geq 130 / 85$ & $109(76.8 \%)$ & $18(31.0 \%)$ \\
\hline Triglyceride level $\geq 150$ & $93(65.5 \%)$ & $6(10.3 \%)$ \\
\hline $\begin{array}{l}\text { Low HDL-C } \\
\text { Increased waist } \\
\text { circumference }\end{array}$ & $110(50.7 \%)$ & $5(8.6 \%)$ \\
\hline $\begin{array}{l}\text { Known case of } \\
\text { hypertension }\end{array}$ & $86(60.6 \%)$ & $14(24.1 \%)$ \\
\hline \begin{tabular}{l} 
History of cardiac disease \\
\hline
\end{tabular} & $24(16.9 \%)$ & $10(17.2 \%)$ \\
\hline
\end{tabular}

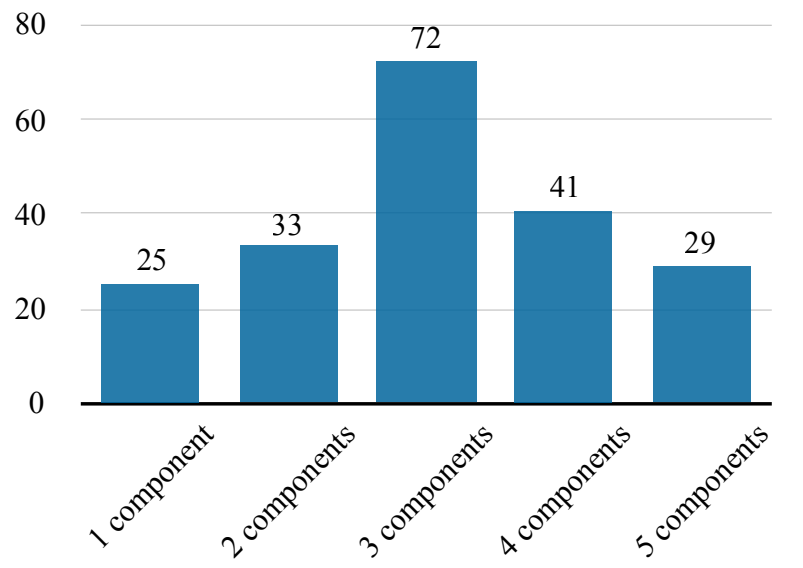

Figure 1. Frequency of components of metabolic syndrome among 200 diabetic patients

years had more than three components of metabolic syndrome (Figure 2).

\section{DISCUSSION}

The prevalence of metabolic syndrome amongst the patients in our study was $71 \%$. The prevalence worldwide ranges from $45.8 \%$ and $96.3 \% .^{11-12}$ Similar study done at diabetes centre in Galle, Sri Lanka showed the prevalence of metabolic syndrome in Type $2 \mathrm{DM}$ population to be $70 \%$ which was consistent with our study. ${ }^{13}$

Our study showed 84 male and 58 female participants had metabolic syndrome which was not similar to the study by Ford et al. ${ }^{4}$ and Nsiah K et al. ${ }^{14}$ which showed higher prevalence of metabolic syndrome in females. This variation may be due to less number of female patients who approached for hospital services during the study period.

There was high prevalence of central obesity in diabetic patients in the study group, accounting for $77.5 \%$. Similar result was seen in the study done at regional diabetes centre in Galle, Southern Sri Lanka, which showed raised waist circumference as an important predictor for metabolic syndrome defined by all three World Health Organisation (WHO), NCEP-ATP III, and International Diabetes Federation (IDF). ${ }^{13}$ Increased waist circumference is a strong indicator of central obesity and is a health risk factor. Specifically, waist circumference (WC) provides a simple and practical anthropometric measure for assessing central 


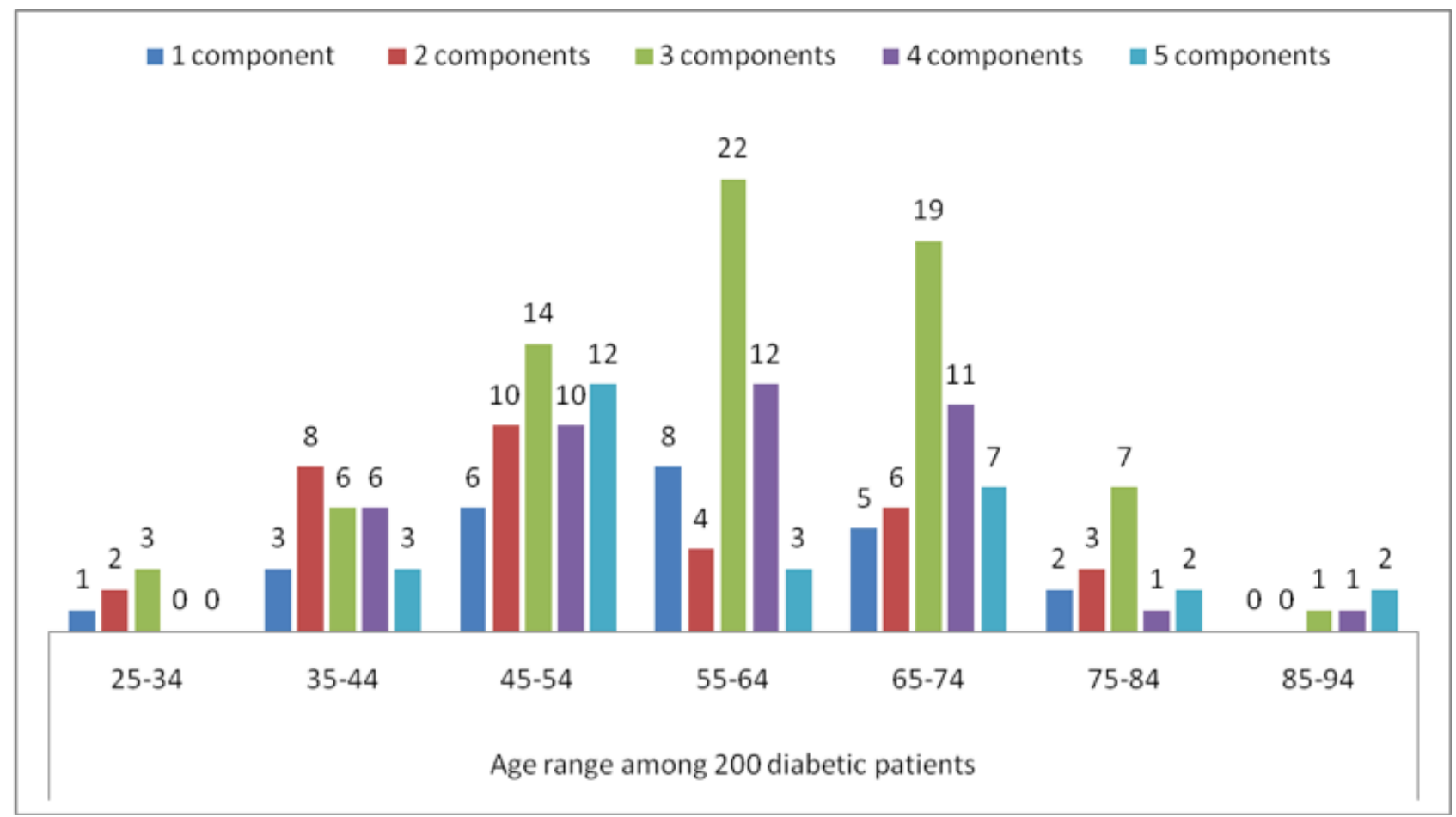

Figure 2. Bar diagram showing the distribution of components of metabolic syndrome according to different age groups

adiposity, and an increasing number of studies are reporting strong associations between $\mathrm{WC}$, visceral adipose tissue, and obesity-related health risks. ${ }^{15}$

Hepatic production of very low density lipoprotein with consequent release of high TGR in blood is associated with insulin resistance resulting from central obesity. ${ }^{16}$ This is consistent with our study which showed more than $50 \%$ of the participants had low HDL. Hypertension was found to be the other commonest component $(76.8 \%)$ in the participating population. This result corresponds to the study done by Nsiah K et al. ${ }^{14}$

In our study $25(12.5 \%)$ patients had at least one component of metabolic syndrome and $33(16.5 \%)$ had two components. This group of population probably suffers from the syndrome within a short period if timely interventions are not taken and lifestyle is not changed. The number of components of metabolic syndrome also shows evidence in the risk of development of cardiovascular diseases (CVD). It also helps to establish interventions and to plan strategies to prevent CVD in this population. ${ }^{17}$ Additionally, in our study 70 (35\%), $41(20.5 \%)$ and $29(14.5 \%)$ of the patients had three, four or five altered components of metabolic syndrome respectively, which were higher than those found among diabetics in other study. ${ }^{17}$

Age groups 45-54, 55-64 and 65-74 years who had more than three components of metabolic syndrome were $14(20 \%), 20(28.6 \%)$ and 19 (27.1\%) respectively, which indicates that the participants of the age group 45-54 years are likely to have a high prevalence of metabolic syndrome, irrespective of sex. Hence, prevention and control measures should be taken when the age approaches since the prevalence of metabolic syndrome increases with age. ${ }^{18}$

\section{CONCLUSIONS}

The prevalence of metabolic syndrome was $71 \%$ among the patients with type 2 diabetes mellitus. It also showed that the prevalence of metabolic syndrome increases with advancing age. The most prevalent component was central obesity and hypertension, followed by, hypertriglyceridemia and low HDL-C. 


\section{ACKNOWLEDGEMENTS}

We extend profound sense of gratitude to the study participants and gives the highest gratitude to the medicine unit staffs of our hospital for the support during research.

To cite this article: Shakya D, KC V. Prevalence of metabolic syndrome in a patients with type 2 diabetes mellitus in a tertiary care hospital. MJSBH. 2019;18(2):36-41.

Conflict of Interest: None declared

\section{REFERENCES}

1. Expert panel on detection, evaluation, and treatment of high blood cholesterol in adults. Executive summary of the third report of the National Cholesterol Education Program (NCEP) Expert Panel on detection, evaluation, and treatment of high blood cholesterol in Adults (Adult Treatment Panel III). JAMA. 2001;285(19):2486-97.

DOI:10.1001/jama.285.19.2486.

2. Manson J, Skerrett P, Greenland P, VanItallie T. The escalating pandemics of obesity and sedentary lifestyle. Arch Intern Med. 2004;164(3):249.

DOI:10.1001/archinte.164.3.249.

3. Ford E. Prevalence of the metabolic syndrome defined by the international diabetes federation among adults in the U.S. Diabetes Care. 2005;28(11):2745-49.

DOI:10.2337/diacare.28.11.2745.

4. Ford E, Giles W, Dietz W. Prevalence of the metabolic syndrome among US adults. JAMA. 2002;287(3):356-9. DOI:10.1001/jama.287.3.356.

5. Zimmet P, Alberti K, Shaw J. Global and societal implications of the diabetes epidemic. Nature. 2001;414(6865): $782-7$.

DOI: $10.1038 / 414782 \mathrm{a}$.

6. American Diabetes Association. Diagnosis and Classification of Diabetes Mellitus. Diabetes care. 2009;32(1):62-7. DOI:10.2337/dc09-S062.

7. Jianping Ye. Mechanisms of insulin resistance in obesity. Frontiers Medicine. 2013;7(1):14-24. DOI:10.1007/s11684-013-0262-6.

8. Lindsay RS, Howard BV. Cardiovascular risk associated with the metabolic syndrome. Curr Diab Rep. 2004;4(1): 63-8. DOI:10.1007/s11892-004-0013-9.

9. Koh K, Han S, Quon M. Inflammatory markers and the metabolic syndrome. J Am Coll Cardiol. 2005;46(11): 1978-85. DOI:10.1016/j.jacc.2005.06.082.

10. Grundy S, Cleeman J, Daniels S, Donato K, Eckel R, Franklin B, et al. Diagnosis and management of the metabolic syndrome. Circulation. 2005;112(17):2735-52. DOI: 10.1161/CIRCULATIONAHA.105.169404

11. Yadav D, Mahajan S, Subramanian S, Bisen P, Chung C, Prasad G. Prevalence of metabolic syndrome in Type 2 diabetes mellitus using NCEP-ATPIII, IDF and WHO definition and its agreement in Gwalior Chambal region of central India. Global Journal of Health Science. 2013;5(6):142-55.

DOI:10.5539/gjhs.v5n6p142.

12. Lombo B, Satizábal C, Villalobos C, Tique C, Kattah W. Prevalence of metabolic syndrome in individuals with Type 2 diabetes mellitus. Acta Med Colomb. 2007;32(1):9-15.

DOI:10.1590/0034-7167-2016-0145. 
13. Nsiah K, Shang V, Boateng K, Mensah F. Prevalence of metabolic syndrome in type 2 diabetes mellitus patients. Int J App Basic Med Res. 2015;5(2):133-8.

DOI:10.4103/2229-516x.157170.

14. Herath HMM, Weerasinghe NP, Weerarathna TP, Amarathunga A. A comparison of the prevalence of the metabolic syndrome among Sri Lankan patients with Type 2 diabetes mellitus using WHO, NCEP-ATP III, and IDF Definitions. Int J Chronic Dis. 2018. 2018.1-8.

DOI:10.1155/2018/7813537.

15. Shen W, Punyanitya M, Chen J, Gallagher D, Albu J, Pi-Sunyer X, et al. Waist circumference correlates with metabolic syndrome indicators better than percentage fat. Obesity. 2006;14(4):727-36. DOI:10.1038/oby.2006.83.

16. Robert CK, Hevener AL, Barnard RJ. Metabolic syndrome and insulin resistance: Underlying causes and modification by exercise training. Compr Physiol. 2013;3(1):1-58. DOI:10.1002/cphy.c110062.

17. Lira Nate JCG, Almeida Xavier Md, Pereira Borges JW ,Maura de Aratijo MF, Coelho Damasceno MM, Freire de Freitas RWJ. Prevalence of metabolic syndrome in individuals with Type 2 Diabetes Mellitus. Revista Brasileira de Enfermagem. 2017;70(2). DOI: 10.1590/0034-7167-2016-0145.

18. Gharipour M, Sadeghi M, Hosseini M, Andalib E, Boroujeni MB, Sarrafzadegan N. Effect of age on the phenotype of metabolic syndrome in developing country. Adv Biomed Res. 2015;4:103.

DOI:10.4103/2277-9175.157796. 